\title{
Determination of gene expression changes of USPIO- labeling on embryonic stem cells
}

\author{
Ka-Wing $\mathrm{Au}^{1}$, Yau-Chi Chan ${ }^{1}$, Hung-Fat Tse ${ }^{1}$ \\ ${ }^{l}$ Department of Medicine, The University of Hong Kong, Hong Kong, China
}

Stem cell therapies are potentially new therapeutic approach for treatment of heart and other diseases. Embryonic stem cells (ESCs) maintain their pluripotency to differentiate into any cell types, which allow the supply of specialized cells. In some animal studies, direct injection of ESCs derived cardiomyocytes after myocardial infarction has been used for repair of myocardial infarct area. Superparamagnetic iron oxide ((U)SPIO) MR imaging have been widely used to monitor the migration of stem cells. This provides a useful tool for noninvasive tracking of cells. Despite of the advantage, the potential functionality change in molecular levels of (U) SPIO-labeled ESCs should be considered. In this present work, we examined the effect of labeling by comparing the gene expression levels, particularly ion channels, of un-labeled and USPIO-labeled mouse ESCs.

Keywords: MR imaging, iron oxide

Cell Research (2008) 18:s127. doi: 10.1038/cr.2008.217; published online 4 August 2008 